\title{
Introduction to the Second International Workshop on Service Oriented Computing in Logistics (SOC-LOG 2010)
}

\author{
Joerg Leukel ${ }^{1, *}$, André Ludwig ${ }^{2, * *}$, and Alex Norta ${ }^{3}$ \\ ${ }^{1}$ Department of Information Systems 2, University of Hohenheim, Stuttgart, Germany \\ joerg.leukel@uni-hohenheim.de \\ ${ }^{2}$ Information Systems Institute, University of Leipzig, Germany \\ ludwig@wifa.uni-leipzig.de \\ ${ }^{3}$ Department of Computer Science, University of Helsinki, Finland \\ alexander.norta@cs.helsinki.fi
}

\begin{abstract}
The purpose of the SOC-LOG workshop was to present and discuss recent significant developments at the intersection of service-oriented computing and logistics systems/supply chain management, and to promote crossfertilization and exchange of ideas and techniques between these fields. The relation to ICSOC 2010 is that, on one hand, the conference addresses the core concepts such as interacting business processes, service composition, service operations, and quality of services, and on the other hand, would receive feedback, experiences, and requirements from a highly relevant application domain to validate and advance its current approaches. The technical program consisted of 4 full papers, 1 position paper (all being reviewed, with an acceptance rate of $56 \%)$, and 1 invited talk.
\end{abstract}

Keywords: logistics, service oriented computing, supply chain management.

\section{Aims and Scope}

Logistics is of paramount importance for many industries: It plans and realizes the flow of goods from sources to destinations by means of transformations in space, time, and quantity. Coordinating logistics activities faces organizational and technical boundaries of the participating firms as well as must resolve conflicting goals and strategies of such firms. Information plays a crucial role in logistics, in particular in today's business environment, which is changing significantly due to, e.g., globalization of supply chains, stronger customer orientation and individualization, all increasing the need for more adaptive logistics systems. IT is the key enabler for managing these challenges, supporting supply chain collaboration, and managing increasing economic dynamics. Advanced IT support allows supply chains to increase their

\footnotetext{
* Funding received by the German Ministry of Education and Research under the project InterLogGrid (BMBF 01IG09010E).

** Funding received by the German Ministry of Education and Research under the projects InterLogGrid (BMBF 01IG09010F) and Logistics Service Bus (BMBF 03IP504).
} 
efficiency significantly, to better fulfill customer needs, and handle the growing organizational complexity and the associated supply chain risks.

While existing logistics IT systems provide solid support for static, self-contained logistics systems, the research on managing the logistics in supply chains that are dynamically changing, is still less advanced. Service oriented computing (SOC) is a promising paradigm, which automates inter-organizational processes with loosely coupled software-based services. The focus of this workshop is the study and exploration of the SOC's potential to solve coordination problems in logistics systems and supply chains.

Logistics systems and supply chains have been subject to much research in Computer Science, Information Systems, and most recently Service Science. Of particular interest are works on adopting ideas, concepts, models, and methods from software technology which follows the service paradigm, i.e., Grid Computing, Serviceoriented Computing, and Cloud Computing. One can identify two major research streams: The first stream is adopting the service paradigm for logistics and supply chain management software. This approach aims at providing the functionality by an integrated set of (Web) services. The second stream is adopting the service paradigm for representing logistics systems and supply chain - or parts of it - by (Web) services. In this sense, the electronic representations become the subject of Web service coordination and its respective body of knowledge.

Key research questions are: (1) How to represent logistics systems in service-based computing systems by employing and adopting constructs, models, and methods of the SOC technology stack, (2) how to describe software-based logistics services with service description languages, (3) how to coordinate software-based logistics services, by employing and adopting approaches for service discovery and service composition, (4) how to negotiate and agree about the delivery of software-based logistics services with approaches for SLA representation, SLA management, and SLA negotiation, and (5) how to control the delivery and how to measure the efficiency and effectiveness of software-based logistics services? With the set of design principles, architectural models and concepts and last but not least with its existing and growing set of standards, SOC promotes adaptiveness of logistics systems and supply chains, a flexible and reconfigurable provisioning along multiple supply chains, and their efficiency.

All submissions received were single-blind peer reviewed by at least two and up to four members of the international program committee. In total, we received nine submissions from three countries. Based on the review reports, we accepted four full papers and one position paper, which amount to an acceptance rate of $55.6 \%$. We would like to thank the program committee members and authors for all of their hard work and participation in the lively workshop. We hope that SOC-LOG will help exchanging new ideas and networking and sharing ideas. More information on SOCLOG 2010 is available at http://soclog10.wifa.uni-leipzig.de.

\section{Workshop Co-chairs}

Joerg Leukel (University of Hohenheim, Germany)

André Ludwig (University of Leipzig, Germany)

Alex Norta (University of Helsinki, Finland) 


\section{Program Committee}

Witold Abramowicz (Poznan University of Economics, Poland)

Samuil Angelov (TU-Eindhoven, Netherlands)

Marcelo Cataldo (Carnegie Mellon University, USA)

Rik Eshuis (TU-Eindhoven, Netherlands)

Diogo Ferreira (IST - Technical University of Lisbon, Portugal)

Paul Grefen (TU-Eindhoven, Netherlands)

Maria-Eugenia Iacob (University of Twente, Netherlands)

Axel Korthaus (Queensland University of Technology, Australia)

Marek Kowalkiewicz (SAP Research, Australia)

Carlos Müller (University of Sevilla, Spain)

Manuel Resinas (University of Seville, Spain)

Manfred Reichert (Ulm University, Germany)

Toni Ruokolainen (University of Helsinki, Finland)

Jun Shen (University of Wollongong, Australia)

Vijay Sugumaran (Oakland University, USA)

Ingo Weber (University of New South Wales, Australia) 